\title{
CHEMODYNAMIC AND PHOTOMETRIC EVOLUTION OF ELLIPTICAL GALAXIES
}

\author{
J. LOXEN, U. FRITZE - V. ALVENSLEBEN AND K.J. FRICKE \\ Universitäts-Sternwarte Göttingen \\ Geismarlandstraße 11, 37083 Göttingen
}

\author{
AND \\ G. HENSLER \\ Universitätssternwarte Kiel \\ Olshausenstraße 40, $24118 \mathrm{Kiel}$
}

In many hydrodynamical models of elliptical galaxies the absence of information about the detailed evolution of stars and the spectro-photometric output by hot gas and stars leads to a missing link between the data we obtain in observations (photons) and the internal behavior (dynamics) of a galaxy.

As an approach to a better understanding of this we developed a coupling of two model descriptions for elliptical galaxies. The first is a chemodynamic code which handles the interaction of several star and gas components in an eulerian one-dimensional grid including complete hydrodynamics via equations of state, momentum and energy. Few results about observable features are given by this model. The second description is a one-zone model of galaxies with raw assumptions about internal dynamics but with a detailed scheme for the evolution of stars in a Monte-Carlo method. Luminosities, colours and spectra can be calculated with this code.

The coupling of these two descriptions establishes the missing link: The impact of stellar evolution (e.g. heating by supernovae ...) on the internal dynamics and, vice versa, the influence of hydrodynamics back on observable data (due to star-formation or cooling processes etc.) can now be studied in detail and compared directly with observations.

This work was supported in part by DFG-grant Fr 325/33-1. 